\title{
An Elderly Patient with Diabetic Nephropathy Complicated by ANCA-associated Nephritis
}

\author{
Tomoya Nishino ${ }^{1}$, Kana Minami ${ }^{1}$, Tadashi Uramatsu ${ }^{1,2}$, Yoko Obata ${ }^{1,3}$, Hideyuki Arai ${ }^{1}$, \\ Noriho Sakamoto ${ }^{1}$, Takashi Taguchi ${ }^{4}$ and Shigeru Kohno ${ }^{1}$
}

\begin{abstract}
A 67-year-old man, on oral therapy for type 2 diabetes mellitus since 1990, had sustained proteinuria since 2005. When hematuria was first discovered in 2008, renal dysfunction [creatinine (Cr), $1.2 \mathrm{mg} / \mathrm{dL}$ ], inflammation [C-reactive protein (CRP), $12 \mathrm{mg} / \mathrm{dL}$ ] and high myeloperoxidase anti-neutrophil cytoplasmic antibodies (MPO-ANCA) levels [546 ELISA units (EU)] were observed. Renal biopsy showed the diagnosis of ANCA-associated nephritis combined with diabetic nephropathy. For this patient, there was pathological proof of the combination of diabetic nephropathy and ANCA-associated vasculitis.
\end{abstract}

Key words: ANCA-associated nephritis, type 2 diabetes mellitus, diabetic nephropathy

(Intern Med 51: 1227-1232, 2012)

(DOI: 10.2169/internalmedicine.51.6775)

\section{Introduction}

There has been a steady increase in diabetic nephropathy in recent years, which has been caused by increased numbers of diabetic patients and interruptions in follow-up. Since 1998, diabetic nephropathy has been the primary cause of the initiation of dialysis in Japan; the management of diabetic nephropathy is therefore very important from the standpoint of prognosis. Approximately $20 \%$ to $30 \%$ of diabetic nephropathy cases are also complicated by nondiabetic nephropathy, particularly IgA nephropathy and membranous nephropathy (1). Thus, complication by another nephrotic syndrome should be considered when there is worsening of renal dysfunction or abnormal urinalysis that does not concur with the natural course of diabetic nephropathy.

To date, there have been 9 reported cases of diabetic nephropathy complicated by nephritis and associated with anti-neutrophil cytoplasmic antibodies (ANCAs) (2-9), including the present case, but only 3 of these cases have shown pathological evidence of diabetic nephropathy. The present case is a rare case of nephropathy in which patho- logical examination proved the co-existence of ANCAassociated nephritis and diabetic nephropathy. ANCAassociated nephritis, which is often seen in the elderly, can present with a high rate of rapidly progressive glomerulonephritis (RPGN), leading to renal failure; therefore, early diagnosis and treatment are very important.

In the current case, the patient presented with the pathophysiological characteristics of RPGN during the course of diabetic nephropathy; the pathological findings of a renal biopsy sample confirmed that the patient had diabetic nephropathy in combination with ANCA-associated nephritis.

\section{Case Report}

The patient was a 67-year-old man with primary complaints of hematuria and proteinuria. Although there was nothing noteworthy in the family history, there was a past incident of cerebral infarction in the left basal ganglia in June 2007. The patient had a history of smoking 20 cigarettes a day (from age 20 to 67 years), but no history of drinking alcohol.

Type 2 diabetes mellitus was first noted in 1990, and medication was started. Proteinuria had been seen since

\footnotetext{
${ }^{1}$ The Second Department of Internal Medicine, Nagasaki University School of Medicine, Japan, ${ }^{2}$ Division of Blood Purification, Nagasaki University Hospital, Japan, ${ }^{3}$ Career Development Center for Medical Doctor, Nagasaki University Hospital, Japan and ${ }^{4}$ Department of Pathology, Nagasaki University Graduate School of Biomedical Sciences, Japan

Received for publication October 21, 2011; Accepted for publication January 24, 2012

Correspondence to Dr. Tomoya Nishino, tnishino@nagasaki-u.ac.jp
} 


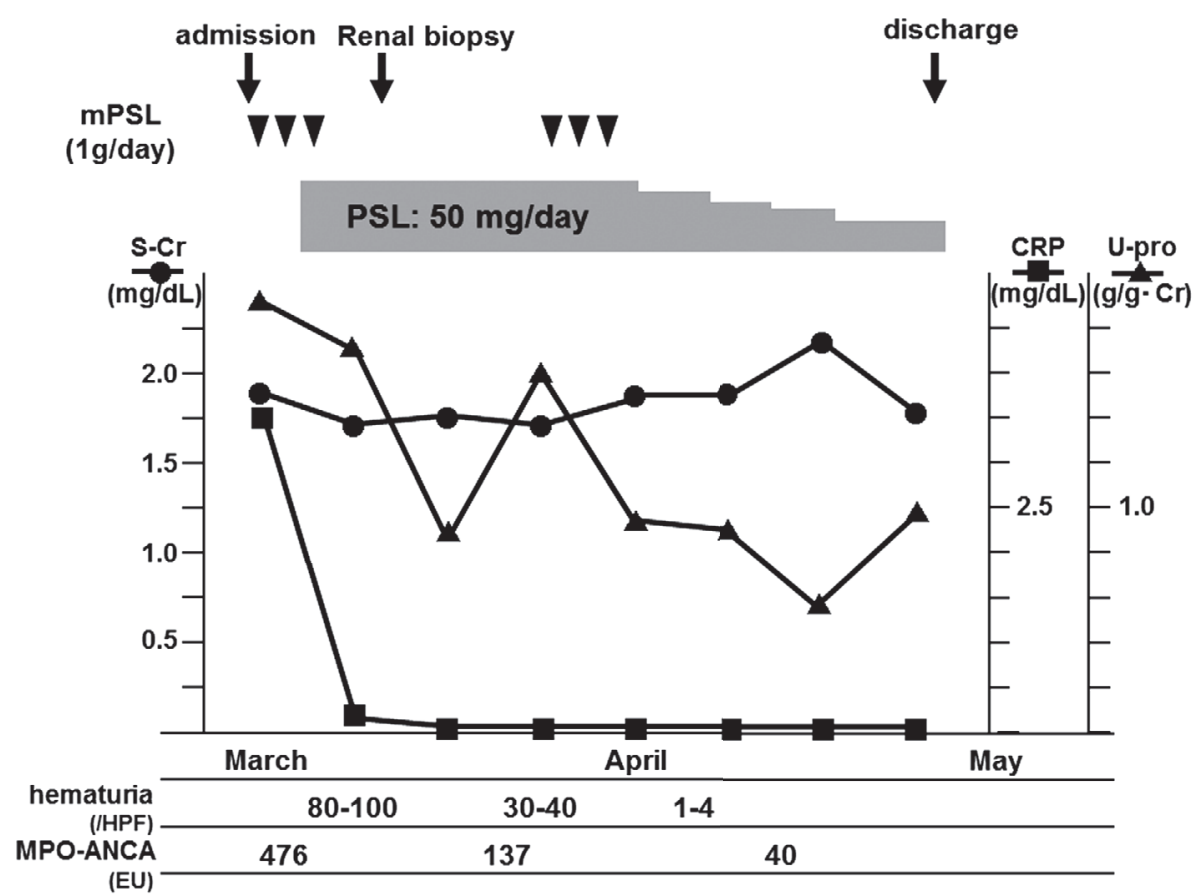

Figure 1. Clinical course

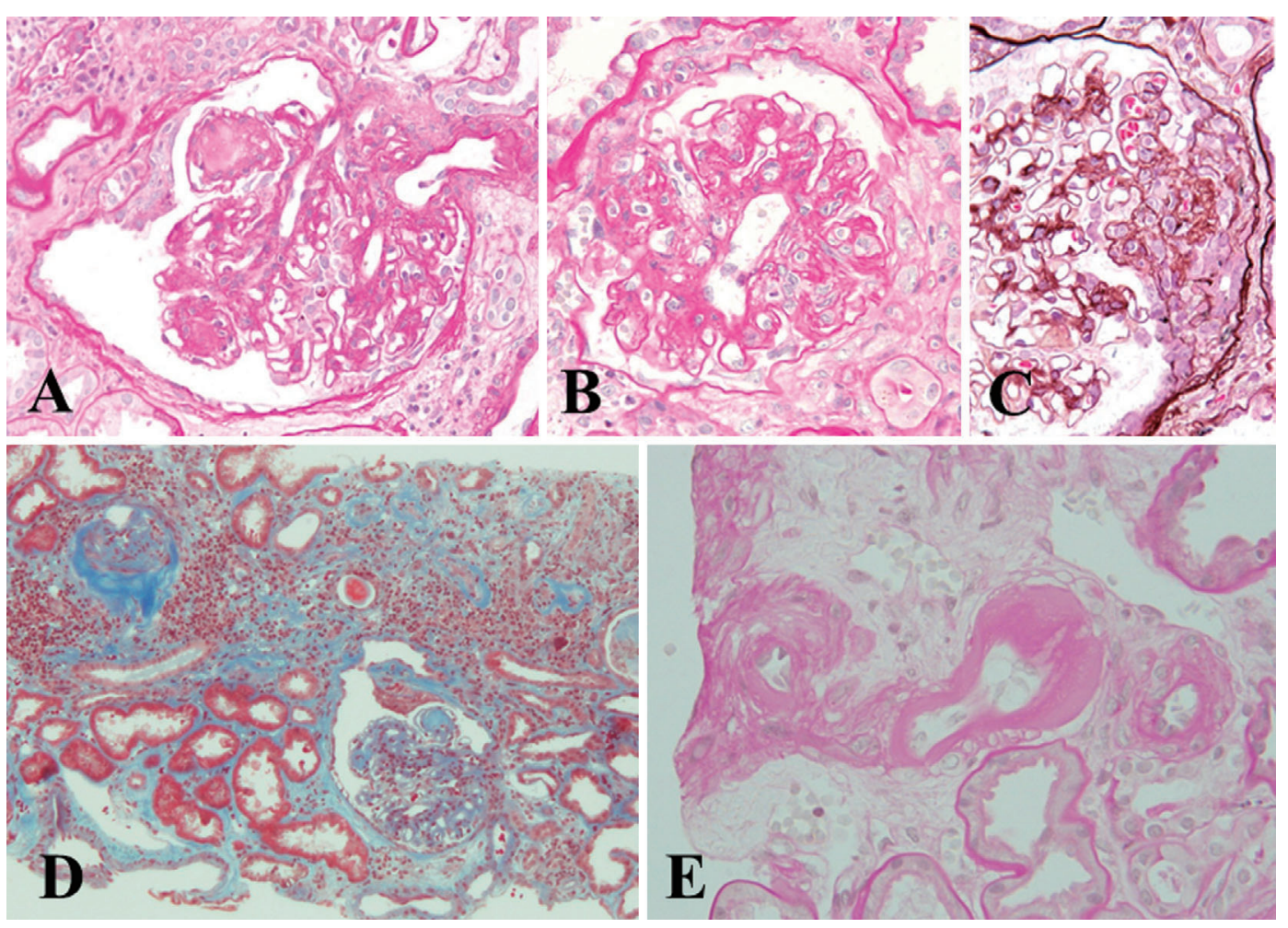

Figure 2. Light microscopy of a renal biopsy sample. A) Nodular lesions characteristic of diabetic glomerulosclerosis are shown (Periodic acid Schiff (PAS) staining, $\times 400$ ). B) and C) A glomerulus with fibrocellular crescent formation is shown (B: PAS staining, C: Periodic acid-methenamine-silver $($ PAM) staining, $\times 400)$. D) Cellular infiltration and fibrosis in interstitium and a sclerotic glomerulus are shown (Masson's trichrome staining $\times 400$ ). E) Hyaline thickening of the arteriole is shown (PAS staining $\times \mathbf{4 0 0})$.

lobular artery and hyaline thickening of the arterioles were also observed (Fig. 2E). Immunofluorescence staining showed only slight deposition of IgM in the mesangial re- gion. Electron microscopy showed diffuse thickening of the glomerular basement membrane and fibrinous dense material in Bowman's space. (Fig. 3). On the basis of the clinical 


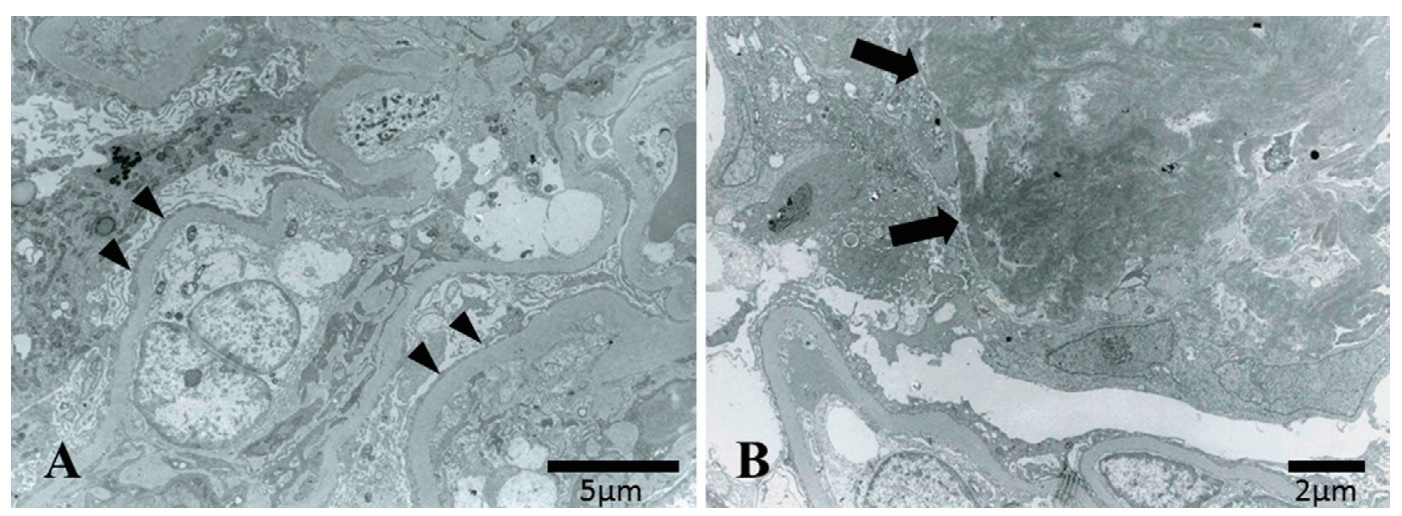

Figure 3. Electron microscopy of a renal biopsy sample. A) The effacement of the foot process is apparent. The glomerular basement membrane is diffusely thickened (arrowheads). B) Fibrinous dense material (arrow) is in Bowman's space. No electron dense deposits are observed.

course and pathological results, the renal dysfunction was diagnosed as diabetic nephropathy complicated by MPOANCA-associated nephritis.

After steroid pulse therapy was started, the inflammation improved (CRP, $0.62 \mathrm{mg} / \mathrm{dL}$ ) by day 4. Furthermore, the MPO-ANCA level decreased to $137 \mathrm{EU}$ by day 21. Additionally, the $\mathrm{Cr}$ was $1.7 \mathrm{mg} / \mathrm{dL}$, and the urinary protein was around 0.5-1.5 g/day, but hematuria persisted at levels of 30-40 RBC/HPF. Since there appeared to be sustained vasculitis activity, steroid pulse treatment was repeated on day 26 after admission (1 g/day of methylprednisolone for 3 days). Although proteinuria persisted at around 0.5-1.0 g/day after the treatment, hematuria was negative, and $\mathrm{Cr}$ was about $1.7-2.0 \mathrm{mg} / \mathrm{dL}$. PSL was administered orally for 1 month at $50 \mathrm{mg} /$ day and then reduced to $30 \mathrm{mg} /$ day after 3 weeks. When the CRP level was less than $0.5 \mathrm{mg} / \mathrm{dL}$, the hematuria was negative, the urinary protein was around 0.5 $\mathrm{g} /$ day, and there was no observed aggravation of the ANCAassociated nephritis, the patient was discharged on day 56 after admission. He was confirmed to be negative for ANCA at an outpatient procedure performed in early July.

During the clinical course, because his blood pressure control was poor with $20 \mathrm{mg}$ /day of nifedipine, telmisartan at a dosage of $20 \mathrm{mg} /$ day was added on day 9 after admission. With regard to diabetes mellitus, despite intensive insulin therapy, his HbA1c level was $9.7 \%$ on admission. As his blood glucose level increased due to corticosteroid therapy, we increased the dose of insulin. As a result, his HbA1c level improved to $8.8 \%$ on day 26 after admission. In addition, pulmonary findings with interstitial pneumonia did not change after steroid therapy.

\section{Discussion}

The incidence of diabetic nephropathy has increased steadily over the past few years. In Japan, it is the disease for which dialysis is most commonly required (approximately $40 \%$ of cases). The prognosis is especially poor for patients receiving dialysis due to the diabetic nephropathy rather than other diseases; the 5-year survival rate for these patients is about $50 \%$. In contrast, in ANCA-associated nephritis, ANCAs, which are observed in the serum, are believed to be involved in the pathogenesis of the nephritis. ANCA-associated nephritis is classified into two types according to the corresponding type of antigen, MPO-ANCAassociated nephritis and PR3-ANCA-associated nephritis. There have been reports of nephritis cases that have tested positive for other types of ANCAs. In Japan, MPO-ANCAassociated nephritis accounts for more than $80 \%$ of the cases, with a predilection for people in their $60 \mathrm{~s}$ and $70 \mathrm{~s}$; people over 80 years of age account for about $10 \%$ of the total number of cases (11).

The frequency of non-diabetic glomerulonephritis with diabetes has been reported to be $22 \%$, and the rate of each type of nephritis with diabetes has been reported as follows: $10.4 \%$ for mesangial proliferative nephritis, $4.9 \%$ for membranous nephropathy, $3.0 \%$ for intratubular proliferative nephritis, $2.4 \%$ for membranous proliferative glomerulonephritis, and $1.2 \%$ for minimal change nephrotic syndrome (12). Clinical differential points for suspecting a new nephritis during the course of diabetic nephropathy include: 1) persistent hematuria of greater than a moderate degree (more than $50 \mathrm{RBC} / \mathrm{HPF}$ ); 2) the appearance of proteinuria or nephrotic syndrome in the early stage of diabetes (5-10 years); 3) rapid deterioration of renal function; and 4) proteinuria or renal failure despite a lack of any other observable microangiopathy (12-16). These findings provide a reason to perform a renal biopsy for differential diagnosis. Both moderate hematuria and rapid deterioration of renal function were applicable in the present case, which had high levels of MPOANCAs on close inspection, and the results of the renal biopsy revealed that the diabetic nephropathy had been complicated by MPO-ANCA-associated nephritis.

There have not been many reports to date of MPOANCA-associated nephritis complicating diabetic nephropathy; 9 case reports were identified in the literature (Table 2). The patients' ages ranged from 50 to 88 years old, with 5 male patients and 4 female patients. The duration of diabetes tended to be long, and all cases exhibited proteinuria and hematuria. Hemodialysis was initiated in 3 of the 9 cases, 
Table 2. Patients Reported to have Diabetic Nephropathy Complicated by ANCAassociated Glomerulonephritis

\begin{tabular}{|c|c|c|c|c|c|c|c|}
\hline No. Age & (years)/Sex & $\begin{array}{l}\text { Duration } \\
\text { (years) }\end{array}$ & $\mathrm{R}$ & $\begin{array}{l}\text { ANCA } \\
(\mathrm{EU})\end{array}$ & $\begin{array}{l}\text { Proteinuria } \\
\text { (g/day) }\end{array}$ & $\begin{array}{l}\text { Hematuria } \\
(/ \mathrm{HPF})\end{array}$ & Outcome \\
\hline$\overline{1^{2)}}$ & $64 \mathrm{M}$ & 16 & $(-)$ & 548 & 1.3 & Many & Improved \\
\hline $2^{3)}$ & $55 \mathrm{~F}$ & 25 & $(+)$ & 531 & 1.5 & $60-80$ & Improved \\
\hline $3^{4)}$ & $78 \mathrm{M}$ & 30 & $(+)$ & 196 & 4.0 & Many & ESRD (HD) \\
\hline $4^{5)}$ & $65 \mathrm{M}$ & 13 & $(-)$ & $8.4(\mathrm{U} / \mathrm{mL})$ & NA & + & ESRD (HD) \\
\hline $5^{6)}$ & $67 \mathrm{~F}$ & 3 & $(+)$ & 240 & 4.2 & 250 & ESRD (HD) \\
\hline $6^{7)}$ & $72 \mathrm{~F}$ & 10 & NA & NA & 5.4 & + & Improved \\
\hline $7^{8)}$ & $50 \mathrm{M}$ & 7 & $(-)$ & 903.5 & & Many & Improved \\
\hline $8^{9)}$ & $88 \mathrm{M}$ & 30 & $(-)$ & 767.2 & & $50-99$ & Died \\
\hline This case & $67 \mathrm{M}$ & 17 & $(+)$ & 546 & 1.9 & $80-100$ & Improved \\
\hline
\end{tabular}

Abbreviations: R\#: reference number, Duration: duration of diabetes mellitus, R: diabetic retinopathy, ANCA: antineutrophil cytoplasmic antibody, ESRD: end-stage renal failure, NA: not available

and 1 case resulted in death.

Accardo-Palumbo et al. in 1996, reported a relationship between diabetes and MPO-ANCA-associated nephritis, and compared to healthy patients, type 1 diabetes mellitus patients had a particularly high rate of MPO-ANCA positivity (17). Chronic activation of polymorphonuclear neutrophils in type 1 diabetes mellitus, which results from the absolute lack of insulin and subsequent deregulation of glucose metabolism, is believed to be the cause, but the details remain unclear (18). The relationship between type 2 diabetes and MPO-ANCA-associated nephritis is unknown.

With the object of pulmonary findings, although the reasons for ineffectiveness of steroid therapy were not clear in the present case, we considered that chronic interstitial change which was resistant to steroid therapy might exist or a smoking-induced interstitial lung abnormality would affect the pulmonary findings. With regard to the relationship between smoking habits and ANCA positivity, there are conflicting reports. One report shows a higher prevalence of smoking in patients with ANCA-associated nephritis (19). On the other hand, there are studies showing no association between smoking status and ANCA-associated vasculitis $(20,21)$. Thus, we could not determine whether the smoking habit influenced ANCA positivity in the present case.

The present case showed increases and nodular lesions of the mesangial matrix, as well as cellular crescents, on light microscopy. Findings from the fluorescent antibody technique confirmed only small $\operatorname{IgM}$ deposits in the mesangial region, a finding consistent with ANCA-associated nephritis with pauci-immunity. Electron microscopy findings showed diffuse thickening of the basal membrane, a characteristic diabetic nephropathy transformation, and fibrinous dense material in Bowman's space, which is characteristic of inflammatory changes caused by ANCA-associated nephritis. Of the 9 reported cases of combined diabetic nephropathy and ANCA-associated nephritis, diabetic nephropathy was pathologically confirmed by electron microscopy in only 3 cases; we believe that confirmation of nephropathy in combination with ANCA-associated nephritis is rare.
We suggest that, even in elderly diabetic patients with a long history of illness, when a rapid deterioration of renal function or a high degree of microscopic hematuria is observed, complication by a renal disease other than diabetic nephropathy must be considered and dealt with promptly, including renal biopsy.

\section{The authors state that they have no Conflict of Interest (COI).}

\section{References}

1. Olsen S, Mogensen C. How often is NIDDM complicated with non-diabetic renal disease? Diabetologia 39: 1638-1645, 1996.

2. Maeda Y, Tomura S, Kato K, et al. Churg-Strauss syndrome associated with necrotizing crescentic glomerulonephritis in a diabetic patient. Intern Med 36: 68-72, 1997.

3. Takeshita Y, Takagi N, Yamada A, Aikawa T, Umemura S. Diabetes mellitus associated with rapidly progressive glomerulonephritis with perinuclear antineutrophil cytoplasm antibodies. Intern Med 39: 154-156, 2000.

4. Ninomiya T, Kanai H, Hirakawa M, et al. Myeloperoxidaseantineutrophil cytoplasmic antibody-associated glomerulonephritis superimposed on biopsy-proven diabetic nephrosclerosis. Am J Kidney Dis 39: 1-6, 2002.

5. Meoro A, Rivera F, Gil CM, et al. Diabetic glomerulosclerosis associated with renal microscopic polyangeitis. Nephron 83: 176177, 1999.

6. Peces R, Rodríguez M, Pobes A, Seco M. Sequential development of pulmonary hemorrhage with MPO-ANCA complicating antiglomerular basement membrane antibody-mediated glomerulonephritis. Am J Kidney Dis 35: 954-957, 2000.

7. Lui SL, Chan KW, Yip PS, et al. Simultaneous occurrence of diabetic glomerulosclerosis, IgA nephropathy, crescentic glomerulonephritis, and myeloperoxidase-antineutrophil cytoplasmic antibody seropositivity in a Chinese patient. Am J Kidney Dis 40: E 14, 2002.

8. Keven K, Akar H, Kutlay S, Nergizoglu G, Erbay B, Erturk S. MPO-ANCA-associated pulmonary-renal vasculitis in a patient with diabetes mellitus. J Nephrol 15: 720-723, 2002.

9. Nishijima R, Araki A, Ando M, et al. Diabetes mellitus complicated with rapidly progressive glomerulonephritis in an elderly patient. Intern Med 44: 1078-1083, 2005.

10. Matsuo S, Imai E, Horio M, et al. Revised equations for estimated GFR from serum creatinine in Japan. Am J Kidney Dis 53: 982992, 2009. 
11. Fujimoto S, Watts RA, Kobayashi S, et al. Comparison of the epidemiology of anti-neutrophil cytoplasmic antibody-associated vasculitis between Japan and the UK. Rheumatology (Oxford) 50: 1916-1920, 2011

12. Chihara J, Takebayashi S, Taguchi T, Yokoyama K, Harada T, Naito S. Glomerulonephritis in diabetic patients and its effect on the prognosis. Nephron 43: 45-49, 1986.

13. Venkateswara K, Crosson JT. Idiopathic membranous glomerulonephritis in diabetes patients. Report of three cases and review of the literature. Arch Intern Med 140: 624-627, 1980.

14. Yum M, Maxwell DR, Hamburger R, Kleit SA. Primary glomerulonephritis complicating diabetic nephropathy. Report of seven cases and review of the literature. Hum Pathol 15: 921-927, 1984.

15. Amoah E, Glickman JL, Malchoff CD, Stergill BC, Kaiser DL, Bolton WK. Clinical identification of nondiabetic renal diseases in diabetic patients with type I and type II disease presenting with renal dysfunction. Am J Nephrol 8: 204-211, 1988.

16. Aziz S, Cohen AH, Winer RL, Llach F, Massry SG. Diabetes mellitus with immune complex glomerulonephritis. Nephron 23: 32-
37, 1979.

17. Accardo-Palumbo A, Triolo G, Giardina E, Carbone MC, Ferrante A, Triolo G. Detection of anti-myeloperoxidase antibodies in the serum of patients with type 1 diabetes mellitus. Acta Diabetol 33: 103-107, 1996.

18. Nasr SH, D'Agati VD, Said SM, et al. Pauci-immune crescentic glomerulonephritis superimposed on diabetic glomerulosclerosis. Clin J Am Soc Nephrol 3: 1282-1288, 2008.

19. Sessa A, Meroni M, Battini G, Vaccari M, Giordano F, Torri Tarelli L. Cigarette smoking and pauci-immune extracapillary glomerulonephritis with ANCA-associated idiopathic systemic vasculitis. Contrib Nephrol 130: 103-108, 2000.

20. Lane SE, Watts RA, Bentham G, Innes NJ, Scott DG. Are environmental factors important in primary systemic vasculitis? A case-control study. Arthritis Rheum 48: 814-823, 2003.

21. Haubitz M, Woywodt A, de Groot K, Haller H, Goebel U. Smoking habits in patients diagnosed with ANCA associated small vessel vasculitis. Ann Rheum Dis 64: 1500-1502, 2005.

(C) 2012 The Japanese Society of Internal Medicine http://www.naika.or.jp/imindex.html 